\title{
形状记忆合金超弹性螺旋弹簧的力学模型
}

\author{
周 博 王志勇 薛世峰 \\ (中国石油大学(华东)储运与建筑工程学院 青岛 266580)
}

\begin{abstract}
摘要: 结合普通金属螺旋弹簧的弹性变形理论和形状记忆合金(SMA)的力学本构模型, 分析与描述 SMA 螺旋弹簧的簧丝横 截面上应变、应力分布规律, 进而推导 SMA 螺旋弹簧的相变临界参数计算公式。基于 SMA 螺旋弹簧的宏观试验现象和推导 的相变临界参数计算公式, 建立描述 SMA 螺旋弹簧的轴向变形和轴向外力间关系的力学模型。理论计算与试验结果的对比 表明, 建立的 SMA 螺旋弹簧力学模型能准确预测 SMA 螺旋弹簧的轴向外力和轴向变形间的关系, 并克服有限单元法模拟计 算 SMA 螺旋弹簧时在几何建模和数值收玫等方面的局限性, 可为研究 SMA 螺旋弹簧的力学行为和基于 SMA 螺旋弹簧的结 构设计提供必要的理论基础和技术参考。
\end{abstract}

关键词: 形状记忆合金; 螺旋弹簧; 相变临界参数; 力学模型

中图分类号: TG139

\section{Mechanical Model for Super-elastic Helical Spring of Shape Memory Alloy}

\section{ZHOU Bo WANG Zhiyong XUE Shifeng}

(College of Pipeline and Civil Engineering, China University of Petroleum, Qingdao 266580)

\begin{abstract}
The distributions of strain and stress in the section of the shape memory alloy (SMA) wire of helical spring are analyzed and described based on the deformation theory of ordinary helical spring and constitutive model of SMA. Then the formulas are derived to calculate the phase transition critical parameters of SMA helical spring. A mechanical model, which expresses the relation of axial force and axial deformation in SMA helical spring, is developed based on the experimental phenomena and the formulas of phase transition critical parameters of SMA helical spring. Results show that the developed mechanical model is able to predict the relation of axial force and axial deformation in SMA helical spring and overcome the limitations of geometric modeling and numerical convergence in the finite element method. Therefor it can serve as the theoretical basis and technical reference for the investigation on the mechanical behaviors of SMA helical spring and the structure design based on SMA helical spring.
\end{abstract}

Key words: shape memory alloy; helical spring; phase transition critical parameter; mechanical model

\section{0 前言}

形状记忆合金(Shape memory alloy, SMA)因具 有独特的超弹性和形状记忆效应, 及耐腐蚀、抗疲 劳、阻尼性好等优势, 在土木、机械、航天等领域 中具有广泛应用前景而备受关注与研究。基于 SMA 的装置或结构能否成功实现预期功能的关 键, 是合理选择 SMA 元件以及正确利用 SMA 元 件的特性。丝状或棒状 SMA 由于结构简单和便于 计算，在不同工程领域中被大量使用。于东等 ${ }^{[1]}$ 利用 SMA 丝的形状记忆效应, 借助齿轮与链条 的传动功能设计了一种旋转驱动装置。SHI 等 ${ }^{[2]}$

* 国家重点研发计划资助项目(2017YFC0307604)。20180521 收到初稿, 20181123 收到修改稿
研究了一种利用 SMA 丝主动驱动的两自由度链 接构件, 并用 PID 方法进行模糊逻辑控制 SMA 两端的电压。邓宗才等 ${ }^{[3]}$ 将具有一定初始应变的 SMA 丝埋入混凝土梁中, 通入电流, 研究 SMA 对混凝土梁的驱动效应。李惠等 ${ }^{[4]}$ 利用 SMA 丝 设计了两种耗能器: 拉伸型和剪刀型, 工作过程 中 SMA 丝始终保持受拉状态。陈金等 ${ }^{[5]}$ 利用超 弹性 SMA 棒设计了一种隔振装置, 用于空间网 架结构的水平向隔振。任文杰等 ${ }^{[6]}$ 设计了一种新 型的自复位 SMA 阻尼器, 同时使用了预应变 SMA 束和非预应变 SMA 束, 具有良好的耗能及 复位功能。

虽然丝状或棒状 SMA 结构简单并便于计算, 但是丝状 SMA 不能承受压缩载荷, 棒状 SMA 在压 缩过程易于失稳, 这给丝状或棒状 SMA 实际应用 
带来很大局限。为克服丝状或棒状 SMA 的上述局 限, 人们将 SMA 加工成各式各样的弹簧用于实际 设计中, 其中应用最广泛的是 SMA 螺旋弹簧。 $\mathrm{KOH}$ 等 ${ }^{[7]}$ 提出了一种欧米伽形状的蠕动爬行机器人, 用 电流对 SMA 螺旋弹簧加热实现主动驱动。

FRANCESCO 等 ${ }^{[8]}$ 研究了一种被动磁-热-机耦合系 统, 该系统中 SMA 弹簧被用于磁流变离合器的开 关控制上。SPEICHER 等 ${ }^{[9]}$ 利用 SMA 螺旋弹簧开发 了两种 SMA 张力/压缩装置, 用于建筑物中的支撑 元件, 表现出良好的阻尼性能。蔡锦荣等 ${ }^{[10]}$ 提出了 一种 SMA 弹簧-轴承滚动隔震装置, 并分析了该装 置对某一实际输电塔结构的隔震效果, 结果证明该 装置的隔震效果良好。刘爱荣等 ${ }^{[11]}$ 通过对 SMA 弹 簧变形的分析, 提出可以改变弹簧刚度影响系统共 振频率进而进行振动控制。贺志荣等 ${ }^{[12]}$ 通过示差量 热仪、拉伸试验等对 NITI 合金弹簧的相变和变形特 性进行了研究。

描述与预测 SMA 螺旋弹簧的力学行为, 是基 于 SMA 螺旋弹簧进行各类功能或结构设计的基础, 具有重要的理论和工程实际意义。TOI 等 ${ }^{[13]}$ 使用全 量拉格朗日方法推导了线性铁木辛柯梁单元的有限 元方程, 并将其程序化, 对 SMA 螺旋弹簧的超弹 性大变形进行了模拟。MIRZAEIFAR 等 ${ }^{[14]}$ 分别用解 析方法和数值方法对 SMA 螺旋弹簧进行了分析, 解析解中三维宏观多晶 SMA 本构被缩减至一维纯 剪切情形, 根据是否考虑曲率的影响先后将弹簧簧 杆简化为纯扭转的直杆和曲杆; 数值解中 SMA 三 维本构模型被应用到有限元方法中, 使用实体单元 对 SMA 螺旋弹簧的加卸载过程进行模拟。AGUIAR 等 ${ }^{[15]}$ 对 SMA 螺旋弹簧分别进行了建模、模拟和试 验分析, 首先用一维本构模型对 SMA 热力学剪切 行为进行描述, 接着利用线弹性弹簧的经典分析方 法对 SMA 螺旋弹簧进行建模, 并开发了一种基于 算子分割技术的数值方法对 SMA 螺旋弹簧进行模 拟。HEIDARI 等 ${ }^{[16]}$ 研究了 SMA 螺旋弹簧制备中不 同升角和退火温度对材料热力学特性的影响, 并且 基于 Mises 等效应力和应变, 使用扩展的一维本构 模型对 SMA 螺旋弹簧内的切应力-应变响应进行描 述分析, 研究了几何参数对 SMA 螺旋弹簧轴向拉 力和位移响应的影响。庄鹏等 ${ }^{[17-18]}$ 基于 LIANG 和 ROGERS 提出的 SMA 本构模型, 建立了 SMA 螺旋 弹簧简化力学模型, 对 SMA 螺旋弹簧的力学行为 进行了数值模拟计算。

为准确描述 SMA 螺旋弹簧的力学行为, 根据 普通螺旋弹簧的弹性变形理论和 SMA 力学本构模 型, 研究了 SMA 螺旋弹簧的簧丝横截面上的应变
和应力分布规律, 进而建立了 SMA 螺旋弹簧的相 变临界参数计算公式。基于 SMA 螺旋弹簧的宏观 试验现象和相变临界载荷参数公式, 建立了描述 SMA 螺旋弹簧轴向变形和轴向外力间关系的 SMA 螺旋弹簧力学模型。结合建立的 SMA 螺旋弹簧的 力学模型和相变临界参数公式, 对多种载荷工况下 SMA 螺旋弹簧力学行为进行了数值模拟计算。计算 结果及其与试验结果的对比分析表明, 建立的力学 模型能有效预测 SMA 螺旋弹簧的轴向外力和轴向 变形间的关系, 且便于工程实际应用, 可为研究 SMA 螺旋弹簧的力学行为和基于 SMA 螺旋弹簧的 结构设计提供理论基础和技术参考。

\section{SMA 宏观本构模型}

\section{1 本构方程}

随着 SMA 的本构模型不断被提出 ${ }^{[19-25]}$, 人们 对于形状记忆合金的本构以及相变过程已经有了较 为深入的认识。文献[21-22]结合马氏体体积分数的 变化过程, 将 SMA 的超弹性和形状记忆效应分别 描述为卸载引起的形状记忆效应和加热引起的形状 记忆效应, 并建立了 SMA 的宏观力学本构方程和 细观力学本构方程。在不考虑温度变化的情况下宏 观力学本构方程表示为

$$
\varepsilon_{i j}=\frac{1+v}{E} \sigma_{i j}-\frac{v}{E} \sigma_{k k} \delta_{i j}+\frac{\partial \sigma_{\mathrm{e}}}{\partial \sigma_{i j}} \varepsilon_{\mathrm{L}} \eta
$$

式中, $E$ 为弹性模量、 $\eta$ 为应力诱发马氏体体积分 数、 $\alpha$ 为热膨胀系数、 $\varepsilon_{\mathrm{L}}$ 为 $\mathrm{SMA}$ 最大恢复应变, 可由拉伸试验测定。应力诱发马氏体体积分数 $\eta$ 和 总的马氏体体积分数 $\xi$ 的关系为

$$
\xi=\xi_{0}+\left(1-\xi_{0}\right) \eta
$$

式中, $\xi_{0}$ 为马氏体体积分数初值。SMA 的弹性模量 $E$ 可表示为

$$
E=E_{\mathrm{a}}+\left(E_{\mathrm{m}}-E_{\mathrm{a}}\right) \xi
$$

式中, $E_{\mathrm{a}}$ 和 $E_{\mathrm{m}}$ 分别为 SMA 的奥氏体和马氏体的弹 性模量。等效应力 $\sigma_{\mathrm{e}}$ 可表示为

$$
\sigma_{\mathrm{e}}=\sqrt{\frac{3}{2}\left(\sigma_{i j}-\frac{1}{3} \sigma_{k k} \delta_{i j}\right)\left(\sigma_{i j}-\frac{1}{3} \sigma_{k k} \delta_{i j}\right)}
$$

\section{2 相变方程}

为有效建立 SMA 螺旋弹簧的力学模型, 对周 博等 ${ }^{[21-22]}$ 建立的相变方程进行合理的简化, 即将应 力诱发马氏体体积分数与等效应力间非线性关系简 化为线性关系。在马氏体相变过程, 应力诱发马氏 体体积分数的演化过程描述为 


$$
\eta=\eta_{0}+\left(1-\eta_{0}\right) \frac{\sigma_{\mathrm{e}}-\sigma_{\mathrm{ms}}}{\sigma_{\mathrm{mf}}-\sigma_{\mathrm{ms}}}
$$

式中, $\eta_{0}$ 为马氏体相变开始时的应力诱发马氏体体 积分数的初值。在马氏体逆相变过程, 应力诱发马 氏体体积分数的演化过程描述为

$$
\eta=\eta_{0}^{\prime} \frac{\sigma_{\mathrm{e}}-\sigma_{\mathrm{af}}}{\sigma_{\mathrm{as}}-\sigma_{\mathrm{af}}}
$$

式中, $\eta_{0}^{\prime}$ 为马氏体逆相变开始时的应力诱发马氏体 体积分数的初值。在式(5)中, $\sigma_{\mathrm{ms}}$ 和 $\sigma_{\mathrm{mf}}$ 分别称为 马氏体开始应力和马氏体结束应力, 它们和温度 $T$ 的关系为

$$
\left\{\begin{array}{l}
\sigma_{\mathrm{ms}}=\sigma_{\mathrm{crs}}+<T-M_{\mathrm{s}}>C_{\mathrm{M}} \\
\sigma_{\mathrm{mf}}=\sigma_{\mathrm{crf}}+<T-M_{\mathrm{s}}>C_{\mathrm{M}}
\end{array}\right.
$$

式中, $\sigma_{\mathrm{crs}} 、 \sigma_{\mathrm{crf}} 、 C_{\mathrm{M}}$ 为反映马氏体相变过程中临 界应力和临界温度间关系的材料参数, 材料参数 $M_{\mathrm{s}}$ 和 $M_{\mathrm{f}}$ 为马氏体开始温度和马氏体结束温度。在式 (6)中, $\sigma_{\mathrm{as}}$ 和 $\sigma_{\mathrm{af}}$ 分别称为奥氏体开始应力和奥氏体 结束应力, 它们和温度 $T$ 的关系为

$$
\left\{\begin{array}{l}
\sigma_{\mathrm{as}}=C_{\mathrm{A}}\left(T-A_{\mathrm{s}}\right) \\
\sigma_{\mathrm{af}}=C_{\mathrm{A}}\left(T-A_{\mathrm{f}}\right)
\end{array}\right.
$$

式中, $C_{\mathrm{A}}$ 为反映马氏体逆相变过程中临界应力和临 界温度间关系的材料参数, 材料参数 $A_{\mathrm{s}}$ 和 $A_{\mathrm{f}}$ 为奥氏 体开始温度和奥氏体结束温度。此外, 在式(7)中, 跳跃函数 $<T-M_{\mathrm{s}}>$ 的含义如下

$$
<T-M_{\mathrm{s}}>= \begin{cases}0 & T \leqslant M_{\mathrm{s}} \\ 1 & T>M_{\mathrm{s}}\end{cases}
$$

\section{SMA 螺旋弹簧力学模型}

\section{1 应变分析}

图 1 为受轴向拉力 $F$ 作用的螺旋弹簧, 当螺旋 弹簧的螺旋角 $\theta$ 很小时, 可以假设簧丝横截面与弹 簧轴线在同一平面内。簧丝横截面的内力包括剪力

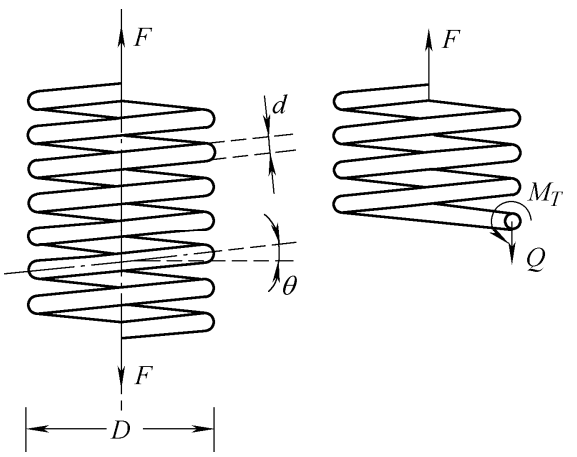

图 1 螺旋弹簧的内力分析图
$Q$ 和扭矩 $M_{\mathrm{T}}$ ，根据平衡关系，可以得到

$$
\left\{\begin{array}{l}
Q=F \\
M_{\mathrm{T}}=\frac{1}{2} F D
\end{array}\right.
$$

式中, $D$ 为弹簧圈的平均直径, $F$ 为螺旋弹簧受到 的轴向拉力。

若假设剪力 $Q$ 引起的切应力在簧丝横截面内均 匀分布, 如图 $2 \mathrm{a}$ 所示, 则剪力 $Q$ 引起的切应力为

$$
\tau_{Q}=\frac{4 F}{\pi d^{2}}
$$

根据线弹性圆轴扭转理论, 在线弹性范围内扭矩 $T$ 在簧丝横截面内引起切应力分布情况如图 $2 \mathrm{~b}$ 所示, 簧丝横截面内的最大切应力为

$$
\tau_{\max }=\frac{8 F D}{\pi d^{3}}
$$

根据式(11)、(12)，可知

$$
\frac{\tau_{Q}}{\tau_{\max }}=\frac{d}{2 D}
$$

根据式(13)可知, 当弹簧丝横截面直径 $d$ 远小于弹 簧圈的平均直径 $D$ 时, 可以忽略剪力 $Q$ 对横截面上 切应力的影响, 进而将弹簧丝内各点应力状态假设 为纯剪切状态, 簧丝横截面上的切应变分布情况如 图 3 所示。

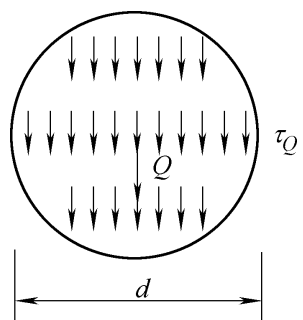

(a)

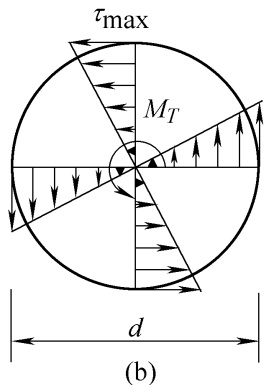

(b)

图 2 弹性范围扭矩引起的切应力分布图

对于线弹性螺旋弹簧, 根据式(12)可知, 簧丝 横截面上的最大切应变为

$$
\begin{gathered}
\gamma_{\text {max }}=\frac{\tau_{\text {max }}}{G}=\frac{8 F D}{\pi G d^{3}} \\
G=\frac{E}{2(1+v)}
\end{gathered}
$$


为切变模量。根据变形能原理, 并利用式(11), 可 以得到螺旋弹簧轴向伸长量 $\lambda$ 和轴向拉力 $F$ 间的 关系为

$$
F=\frac{G d^{4}}{8 D^{3} N} \lambda
$$

式中, $N$ 为螺旋弹簧的总圈数。将式(16)代入式(14), 得到

$$
\gamma_{\max }=\frac{d}{\pi D^{2} N} \lambda
$$

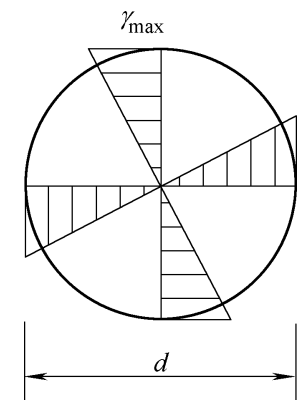

图 3 簧丝横截面上切应变分布图

对于 SMA 螺旋弹簧, 假设其簧丝横截面上的 剪应变仍满足图 3 所示的分布规律。因此 SMA 螺 旋弹簧的簧丝横截面上的最大切应变 $\gamma_{\text {max }}$ 和弹簧轴 向伸长量 $\lambda$ 之间满足式(17)所描述的关系。

\section{2 应力分析}

根据空间应力状态下 SMA 本构方程式(1)并利 用式(12), 可将纯剪切状态下 SMA 本构方程表示为

$$
\gamma=\frac{\tau}{G}+\frac{E}{\sqrt{3} G} \varepsilon_{\mathrm{L}} \eta
$$

式中, $\tau$ 和 $\gamma$ 分别为切应力和工程切应变。根据空 间应力状态下 SMA 相变方程式(5)、(6), 可以得到 纯剪切状态下 SMA 相变方程。在马氏体相变过程,
马氏体体积分数和切应力的关系表示为

$$
\eta=\eta_{0}+\left(1-\eta_{0}\right) \frac{\tau-\tau_{\mathrm{ms}}}{\tau_{\mathrm{mf}}-\tau_{\mathrm{ms}}}
$$

式中, $\tau_{\mathrm{ms}}$ 和 $\tau_{\mathrm{mf}}$ 分别为马氏体开始切应力和马氏体 结束切应力。在马氏体逆相变过程, 马氏体体积分 数和切应力的关系表示为

$$
\eta=\eta_{0}^{\prime} \frac{\tau-\tau_{\mathrm{af}}}{\tau_{\mathrm{as}}-\tau_{\mathrm{af}}}
$$

式中， $\tau_{\mathrm{as}}$ 和 $\tau_{\mathrm{af}}$ 分别称为奥氏体开始切应力和奥氏 体结束切应力。在式(19)中的马氏体开始切应力和 马氏体结束切应力, 分别用前文提到的马氏体开始 应力和马氏体结束应力表示为

$$
\left\{\begin{array}{l}
\tau_{\mathrm{ms}}=\frac{\sqrt{3}}{3} \sigma_{\mathrm{ms}} \\
\tau_{\mathrm{mf}}=\frac{\sqrt{3}}{3} \sigma_{\mathrm{mf}}
\end{array}\right.
$$

在式(20)中的奥氏体开始切应力和奥氏体结束切应 力, 分别用前文提到的奥氏体开始应力和奥氏体结 束应力表示为

$$
\left\{\begin{array}{l}
\tau_{\text {as }}=\frac{\sqrt{3}}{3} \sigma_{\text {as }} \\
\tau_{\text {af }}=\frac{\sqrt{3}}{3} \sigma_{\text {af }}
\end{array}\right.
$$

根据纯剪切状态下 SMA 的本构方程式(18)和相变 方程式(19)、(20), 可以得到不同阶段超弹性 SMA 螺旋弹簧横截面上的切应力分布情况。在加载过程 中切应力分布情况如图 4 所示, 其中, 当 $\tau_{\text {max }} \leqslant \tau_{\mathrm{ms}}$ 时 切应力分布情况如图 $4 \mathrm{a}$ 所示, 当 $\tau_{\mathrm{ms}}<\tau_{\text {max }} \leqslant \tau_{\mathrm{mf}}$ 时 切应力分布情况如图 $4 \mathrm{~b}$ 所示, 当 $\tau_{\text {max }}>\tau_{\mathrm{mf}}$ 时切应 力分布情况如图 4c 所示。
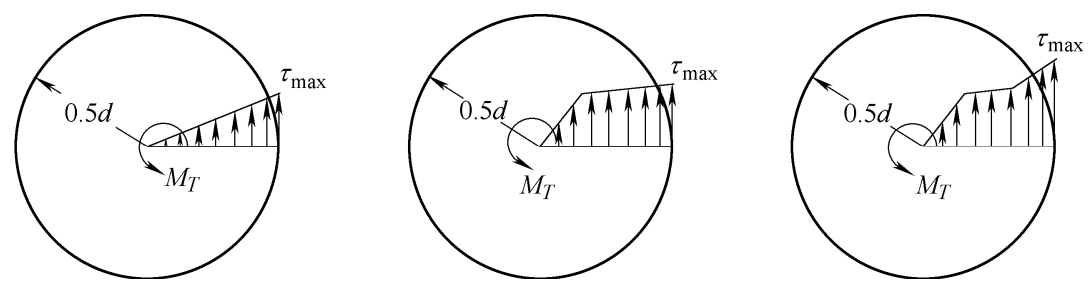

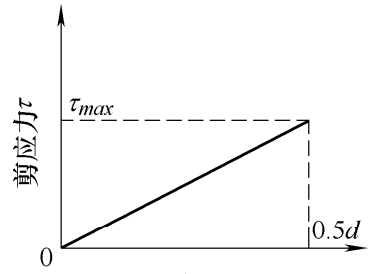

极径 $\rho$

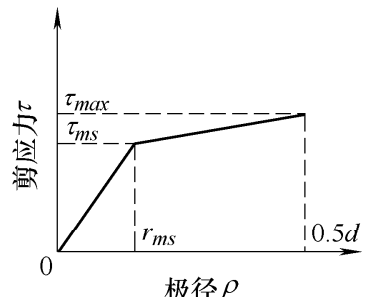

(b)

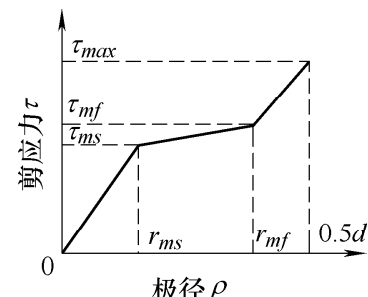

(c)

图 4 加载过程中 SMA 弹簧丝横截面切应力分布情况 


\section{3 相变临界参数}

根据图 4 可以得到, 簧丝横截面扭矩 $M_{\mathrm{T}}$ 和切 应力 $\tau$ 的关系可表示为

$$
M_{T}=2 \pi \int_{0}^{\frac{d}{2}} \tau \rho^{2} \mathrm{~d} \rho
$$

式中, $\rho$ 为簧丝横截面上任一点到其圆心的距离, $d$ 为簧丝横截面的直径。根据式(23)及簧丝横截面扭 矩 $M_{\mathrm{T}}$ 和弹簧轴向外力 $F$ 间的关系式(11), 可以得到

$$
F=\frac{4 \pi}{D} \int_{0}^{\frac{d}{2}} \tau \rho^{2} \mathrm{~d} \rho
$$

式中, $D$ 为螺旋弹簧的平均直径。

如图 4a 所示在 $\tau_{\text {max }} \leqslant \tau_{\mathrm{ms}}$ 情况下, 在加载过程中 簧丝横截面上的最大切应变可表示为

$$
\gamma_{\text {max }}=\frac{\tau_{\text {max }}}{G}
$$

根据式(17)、(25), 可以将 $\tau_{\text {max }}=\tau_{\mathrm{ms}}$ 所对应的 SMA 螺旋弹簧的轴向变形量表示为

$$
\lambda_{\mathrm{ms}}=\frac{\pi D^{2} N}{G d} \tau_{\mathrm{ms}}
$$

将 $\lambda_{\mathrm{ms}}$ 称为马氏体开始变形。根据式(24)和图 $4 \mathrm{a}$, 可 得到 $\tau_{\text {max }}=\tau_{\mathrm{ms}}$ 时 SMA 螺旋弹簧所受的轴力

$$
F_{\mathrm{ms}}=\frac{\pi d^{3}}{8 D} \tau_{\mathrm{ms}}
$$

将 $F_{\mathrm{ms}}$ 称为马氏体开始轴力。

在图 $4 \mathrm{~b}$ 所示 $\tau_{m s}<\tau_{\text {max }} \leqslant \tau_{m f}$ 情况下, 利用纯剪 切状态 SMA 本构方程式(18), 可将簧丝横截面上的 最大切应变表示为

$$
\gamma_{\text {max }}=\frac{\tau_{\text {max }}}{G}+\frac{E}{\sqrt{3} G} \varepsilon_{\mathrm{L}} \eta
$$

根据式(17)、(28), 可以将 $\tau_{\text {max }}=\tau_{\mathrm{mf}}$ 所对应的 SMA 螺旋弹簧的轴向变形量表示为

$$
\lambda_{\mathrm{mf}}=\frac{\pi D^{2} N}{d}\left(\frac{\tau_{\mathrm{mf}}}{G}+\frac{E}{\sqrt{3} G} \varepsilon_{L} \eta\right)
$$

将 $\lambda_{\mathrm{ms}}$ 称为马氏体结束变形。根据式(24)和图 $4 \mathrm{~b}$, 可 得到 $\tau_{\text {max }}=\tau_{\mathrm{mf}}$ 时 SMA 螺旋弹簧所受的轴力

$$
\begin{gathered}
F_{\mathrm{mf}}=\frac{\pi \tau_{\mathrm{ms}}}{D} r_{\mathrm{ms}}^{3}+\frac{\pi a_{1}}{D}\left(\frac{d^{4}}{16}-r_{\mathrm{ms}}^{4}\right)+\frac{4 \pi b_{1}}{3 D}\left(\frac{d^{3}}{8}-r_{\mathrm{ms}}^{3}\right) \\
r_{\mathrm{ms}}=\frac{d}{2 \gamma_{\mathrm{max}}} \gamma_{\mathrm{ms}} \\
\left\{\begin{array}{l}
a_{1}=\frac{\tau_{\mathrm{mf}}-\tau_{\mathrm{ms}}}{\frac{d}{2}-r_{\mathrm{ms}}} \\
b_{1}=\frac{\tau_{\mathrm{ms}} \frac{d}{2}-\tau_{\mathrm{mf}} r_{\mathrm{ms}}}{\frac{d}{2}-r_{\mathrm{ms}}}
\end{array}\right.
\end{gathered}
$$

将 $F_{\mathrm{mf}}$ 称为马氏体结束轴力。

经过与上述类似的分析与推导过程, 卸载过程 中的奥氏体开始变形和奥氏体开始轴力分别可表 示为

$$
\lambda_{\text {as }}=\frac{\pi D^{2} N}{d}\left(\frac{\tau_{\text {as }}}{G}+\frac{E}{\sqrt{3} G} \varepsilon_{\mathrm{L}} \eta\right)
$$

和

$$
\begin{aligned}
& F_{\text {as }}=\frac{\pi \tau_{\text {af }}}{D} r_{\text {af }}^{3}+\frac{\pi a_{1}}{D}\left(\frac{d^{4}}{16}-r_{\text {af }}^{4}\right)+\frac{4 \pi b_{1}}{3 D}\left(\frac{d^{3}}{8}-r_{\text {af }}^{3}\right) \\
& r_{\text {af }}=\frac{d}{2 \gamma_{\max }} \gamma_{\text {af }} \\
& \left\{\begin{array}{l}
a_{2}=\frac{\tau_{\mathrm{as}}-\tau_{\mathrm{af}}}{\frac{d}{2}-r_{\mathrm{af}}} \\
b_{2}=\frac{\tau_{\mathrm{af}} \frac{d}{2}-\tau_{\mathrm{as}} r_{\mathrm{af}}}{\frac{d}{2}-r_{\mathrm{af}}}
\end{array}\right.
\end{aligned}
$$

而卸载过程中的奥氏体结束变形和奥氏体结束轴力 分别表示为

$$
\lambda_{\mathrm{af}}=\frac{\pi D^{2} N}{G d} \tau_{\mathrm{af}}
$$

和

$$
F_{\text {af }}=\frac{\pi d^{3}}{8 D} \tau_{\text {af }}
$$

式(26)、(27)表示的马氏体开始变形和马氏体开 始轴力, 式(29)、(30)表示的马氏体结束变形和马氏 体结束轴力, 式(33)、(34)表示的奥氏体开始变形和 奥氏体开始轴力, 式(37)、(38)表示的奥氏体结束变 形和奥氏体结束轴力, 统称为 SMA 螺旋弹簧的相 变临界参数。SMA 螺旋弹簧的相变临界参数, 是有 效描述 SMA 螺旋弹簧的轴向载荷和轴向变形间定 量关系的重要基础。

\section{4 力学模型}

基于 SMA 螺旋弹簧宏观试验现象和纯剪切状 态下 SMA 本构方程式(18), 可将 SMA 螺旋弹簧轴 向变形 $\lambda$ 和轴向力 $F$ 间关系可描述为

$$
\lambda=\frac{F}{K}+\beta \lambda_{\mathrm{L}}
$$

式中, $K 、 \beta$ 和 $\lambda_{\mathrm{L}}$ 分别称为 SMA 螺旋弹簧的刚度 系数、形状记忆系数和最大记忆变形。SMA 螺旋弹 簧的刚度系数可表示为

$$
K=K_{1}+\left(K_{2}-K_{1}\right) \beta
$$

式中

$$
K_{1}=\frac{F_{\mathrm{ms}}}{\lambda_{\mathrm{ms}}}
$$


为加载过程中线弹性阶段的刚度系数

$$
K_{2}=\frac{F_{\mathrm{mf}}-F_{\mathrm{as}}}{\lambda_{\mathrm{mf}}-\lambda_{\mathrm{as}}}
$$

为卸载过程中线弹性阶段的刚度系数。

在加载过程中, SMA 螺旋弹簧的形状记忆系数 和轴向力间的关系描述为

$$
\beta=\frac{1-\beta_{0}}{2} \cos \left(\pi \cdot \frac{F-F_{\mathrm{ms}}}{F_{\mathrm{ms}}-F_{\mathrm{mf}}}\right)+\frac{1+\beta_{0}}{2}
$$

式中, $\beta_{0}$ 为加载开始时形状记忆系数的初值; 在卸 载过程中, SMA 螺旋弹簧的形状记忆系数和轴向力 间的关系描述为

$$
\beta=\frac{\beta_{0}^{\prime}}{2} \cos \left(\pi \cdot \frac{F-F_{\text {as }}}{F_{\text {af }}-F_{\text {as }}}\right)+\frac{\beta_{0}^{\prime}}{2}
$$

式中, $\beta_{0}^{\prime}$ 为卸载开始时形状记忆系数的初值。

根据式(17), 可将 SMA 螺旋弹簧的最大形状记 忆变形 $\lambda_{\mathrm{L}}$ 和 SMA 的最大残余切应变 $\gamma_{\mathrm{L}}$ 间的关系描 述为

$$
\gamma_{\mathrm{L}}=\frac{d}{\pi D^{2} N} \lambda_{\mathrm{L}}
$$

式中, $D 、 d$ 和 $N$ 分别为 SMA 螺旋弹簧的平均直径、 簧丝横截面直径和圈数。根据单向应力状态的应变 分析, 可将 SMA 的最大残余切应变 $\gamma_{\mathrm{L}}$ 用 SMA 的 最大残余线应变 $\varepsilon_{\mathrm{L}}$ 表示为

$$
\gamma_{\mathrm{L}}=(1+\mu) \varepsilon_{\mathrm{L}}
$$

根据式(45)、(46), 可以将 SMA 螺旋弹簧的最大形 状记忆变形可描述为

$$
\lambda_{\mathrm{L}}=\frac{\pi D^{2} N}{d}(1+\mu) \varepsilon_{\mathrm{L}}
$$

式中, SMA 的最大残余线应变 $\varepsilon_{\mathrm{L}}$ 可以通过拉伸试 验测定。

式(39)、(40)、(43)、(44)、(47)便是建立的描述 SMA 螺旋弹簧轴向力和轴向变形间关系的力学模 型。结合该模型与式(26)、(27)、(29)、(30)、(33)、 (34)、(37)、(38)描述的相变临界参数, 可有效预测不 同工况下 SMA 螺旋弹簧的力学行为, 为基于 SMA 螺旋弹簧的结构设计提供有效的计算与分析方法。

\section{3 算例分析}

利用 SMA 螺旋弹簧的力学模型, 即式(39)、 (40)、(43)、(44)、(47), 并结合相变临界载荷及相 变临界变形计算公式, 即式(26)、(27)、(29)、(30)、 (33)、(34)、(37)、(38), 对 SMA 螺旋弹簧力学行 为进行了数值模拟计算。为便于和试验结果对比分 析, SMA 弹簧几何参数和材料参数均取自文献[9], 如表 1 所示。在后面的数值模拟计算中, SMA 螺 旋弹簧的马氏体体积分数的初值为 0 。

表 1 SMA 螺旋弹簧的材料和几何参数

\begin{tabular}{cccccc}
\hline $\begin{array}{c}\text { 奥氏体弹性模量 } \\
E_{\mathrm{a}} / \mathrm{GPa}\end{array}$ & $\begin{array}{c}\text { 马氏体弹性模量 } \\
E_{\mathrm{m}} / \mathrm{GPa}\end{array}$ & $\begin{array}{c}\text { 马氏体开始相变应力 } \\
\sigma_{\mathrm{ms}} / \mathrm{MPa}\end{array}$ & $\begin{array}{c}\text { 马氏体结束相变应力 } \\
\sigma_{\mathrm{mf}} / \mathrm{MPa}\end{array}$ & $\begin{array}{c}\text { 奥氏体开始相变应力 } \\
\sigma_{\mathrm{as}} / \mathrm{MPa}\end{array}$ & $\begin{array}{c}\text { 奥氏体结束相变应力 } \\
\sigma_{\mathrm{af}} / \mathrm{MPa}\end{array}$ \\
\hline 65.72 & 37.51 & 128.21 & 630.14 & 377.51 & 74.12 \\
\hline \hline 泊松比 $\mu$ & 最大残余应变 $\varepsilon_{\mathrm{L}}$ & 弹簧直径 $D / \mathrm{mm}$ & 簧丝直径 $d / \mathrm{mm}$ & 螺旋弹簧圈数 & 弹簧总长度 $L / \mathrm{mm}$ \\
\hline 0.33 & 0.047 & 38.1 & 12.5 & 5 & 129.8 \\
\hline
\end{tabular}

图 5 为 SMA 螺旋弹簧在拉伸-压缩单循环载荷 下的轴向力和轴向变形间的关系曲线, 其载荷顺序 为: (1)拉伸加载; (2)拉伸卸载; (3)压缩加载; (4)压 缩卸载, 且拉伸和压缩时的最大变形量相同。图 5a、 $5 \mathrm{~b}$ 和 $5 \mathrm{c}$ 分别为最大轴向变形为 $15.2 \mathrm{~mm} 、 30.5 \mathrm{~mm}$ 和 $45.7 \mathrm{~mm}$ 情况下的关系曲线, 其中红色实线为 SMA 螺旋弹簧力学模型描述的曲线、黑色虚线为文 献[9]中的试验结果曲线。计算结果和试验结果均表 明, SMA 螺旋弹簧在拉伸加载-卸载过程和压缩加 载-卸载过程中, 都在轴向力-轴向变形曲线中有明 显的滞回环出现, 这是因为超弹性 SMA 合金在加 载过程中由应力诱发马氏体相变形成的马氏体是不 稳定的, 在卸载过程中发生马氏体逆相变又转变为 稳定的奥氏体相。可以看出 SMA 螺旋弹簧力学模
型描述的曲线和文献[9]中的试验结果曲线吻合较 好, 因此建立的力学模型能有效预测 SMA 螺旋弹 簧的力学行为。

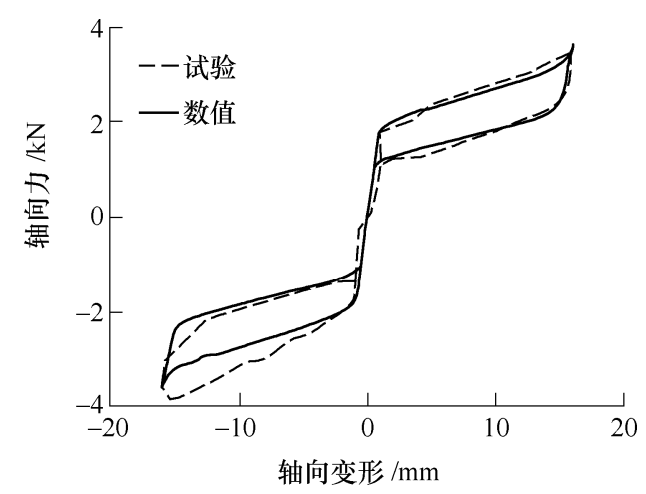

(a) $\lambda_{\text {max }}=15.2 \mathrm{~mm}$ 


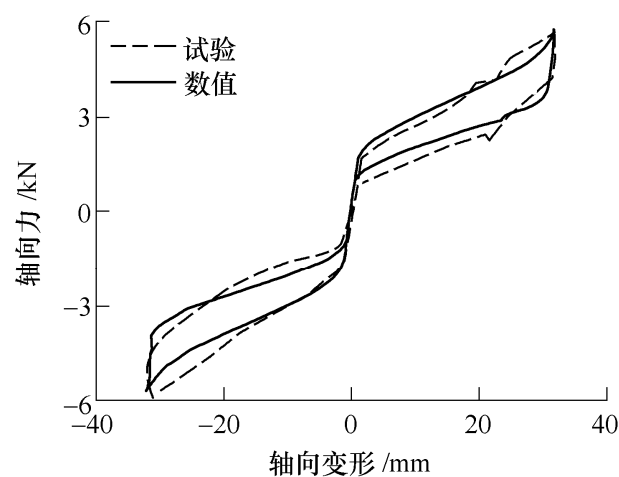

(b) $\lambda_{\max }=30.5 \mathrm{~mm}$

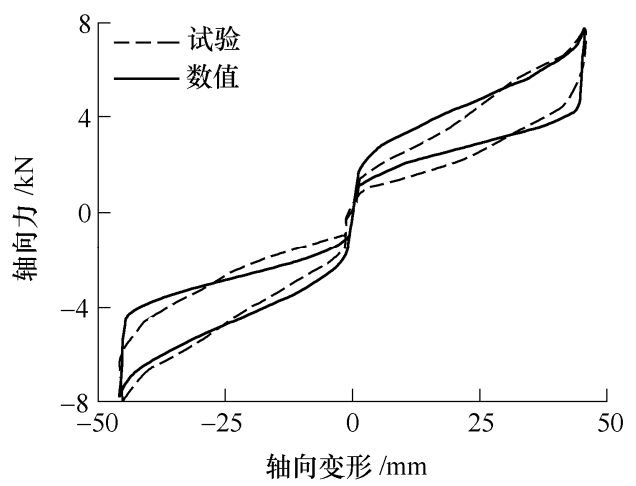

(c) $\lambda_{\max }=45.7 \mathrm{~mm}$

图 5 单循环载荷下 SMA 螺旋弹簧的轴向力与 轴向变形间的关系曲线

为了进一步验证 SMA 螺旋弹簧力学模型的适 用性, 利用其分别对拉伸-压缩多循环复杂载荷情况 和多次往复拉伸复杂载荷情况下 SMA 螺旋弹簧的 力学行为进行了数值模拟计算, 数值模拟中的材料 和几何参数如表 1 所示。图 $6 \mathrm{a}$ 为拉伸-压缩循环复 杂载荷情况的载荷历程曲线, 在载荷循环过程中 SMA 螺旋弹簧的变形幅值逐渐减小; 图 $6 \mathrm{~b}$ 为拉伸压缩循环复杂载荷情况的轴向力与轴向变形关系曲 线, 可以看出在拉伸-卸载和压缩-卸载过程中, 轴 向力-轴向变形曲线中的滞回环均随着 SMA 螺旋弹 簧变形幅值的减小而减小。

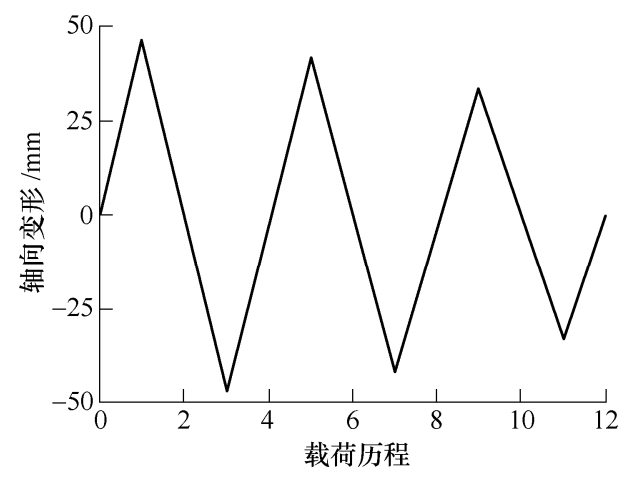

(a) 载荷历程曲线

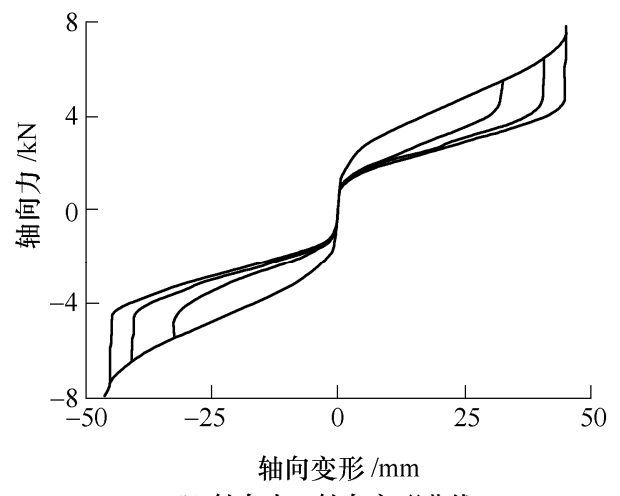

(b) 轴向力 - 轴向变形曲线

图 6 拉伸-压缩多循环复杂载荷情况下 SMA 螺旋弹簧的轴向力-轴向变形曲线

图 7a 为拉伸-循环复杂载荷情况的载荷历程曲 线, 其中第 2 个载荷循环中的变形幅值最小, 且在 第 2 个载荷循环中没有完全卸载就进入第 3 个载荷 循环; 图 $7 \mathrm{~b}$ 为拉伸-循环复杂载荷情况的轴向力与 轴向变形关系曲线, 可以看出在拉伸多循环, 轴向 力-轴向变形曲线中的滞回环也随着 SMA 螺旋弹簧 变形幅值的减小而减小。图 6 和图 7 中的计算结果 表明, 建立的力学模型能够有效描述 SMA 螺旋弹 簧在各种复杂循环载荷作用下的力学行为, 可以为 基于 SMA 螺旋弹簧的各种结构设计提供计算依据, 便于在相关工程领域推广应用。

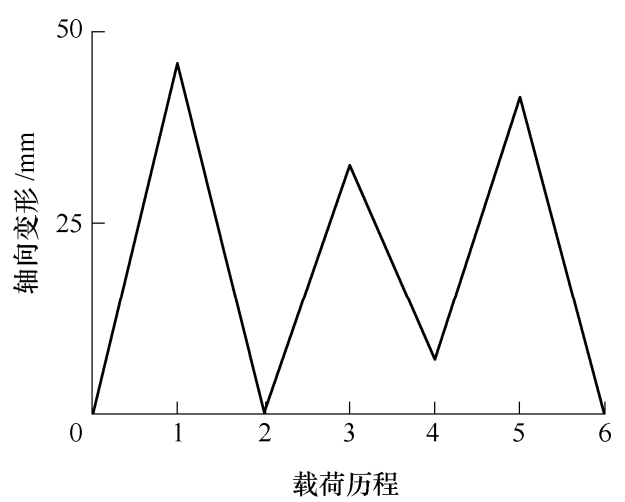

(a) 载荷历程曲线

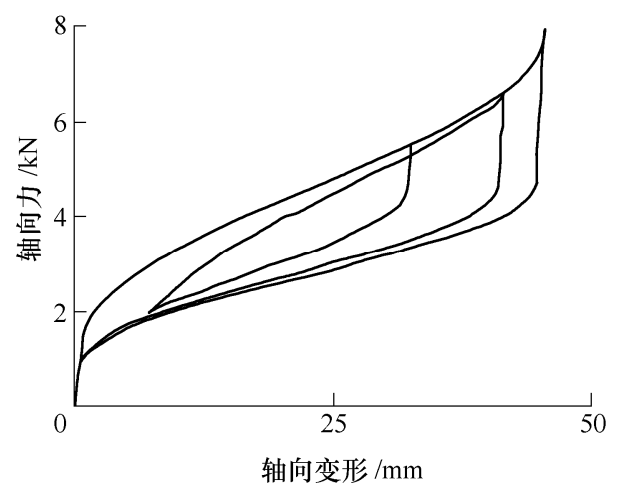

(b) 轴向力 - 轴向变形曲线

图 7 拉伸多循环复杂载荷情况下 SMA 螺旋弹簧的 轴向力-轴向变形曲线 


\section{4 结论}

研究了 SMA 螺旋弹簧的簧丝横截面上应力和 应变分布规律, 建立了描述 SMA 超弹性螺旋弹簧 力学行为的力学模型, 主要结论归纳如下。

(1) 利用普通金属螺旋弹簧弹性变形理论和 SMA 力学本构模型, 描述了 SMA 螺旋弹簧的簧丝 横截面上的应力和应变分布规律。

(2) 根据 SMA 螺旋弹簧的簧丝横截面上的应 力和应变分布规律, 推导了确定 SMA 螺旋弹簧相 变轴力和相变轴向变形的相变临界参数计算公式。

(3) 基于 SMA 螺旋弹簧的宏观试验现象和相 变临界参数计算公式, 建立了描述 SMA 超弹性螺 旋弹簧轴向力和轴向变形间关系的力学模型。

(4) 利用建立的 SMA 螺旋弹簧力学模型计算 了多种工况下 SMA 螺旋弹簧轴向力和轴向变形间 的关系, 计算结果及其与试验结果的对比说明了力 学模型的有效性。

(5) 建立的 SMA 螺旋弹簧力学模型能有效描 述复杂载荷下 SMA 螺旋弹簧的力学行为, 克服了 有限元法在几何建模和数值收玫等方面的局限, 更 便于工程实际应用。

\section{参 考 文 献}

[1] 于东, 张博明, 金龙学, 等. 形状记忆合金旋转驱动器 结构设计方法[J]. 机械工程学报, 2010, 46(14): 91-94. YU Dong, ZHANG Boming, JIN Longxue, et al. Structural design method of rotation gear actuated by shape memory alloys[J]. J. Mech. Eng., 2010， 46(14): 91-94.

[2] SHI Z, WANG T, LIU D, et al. A fuzzy PID-controlled SMA actuator for a two-DOF joint [J]. Chin. J. Aeronaut., 2014, 27(2): 453-460.

[3] 邓宗才, 李庆斌. 形状记忆合金对混凝土梁驱动效应分 析[J]. 土木工程学报，2002，35(2): 41-47.

DENG Zongcai，LI Qingbin. Actuating effects of embedded shape memory alloy on fiber reinforced polymer beam[J]. Chin. Civ. Eng. J., 2002， 35(2): 41-47.

[4] 李惠, 毛晨曦. 新型 SMA 耗能器及结构地震反应控制试 验研究[J]. 地震工程与工程振动, 2003, 23(1): 133-139. LI Hu, MAO Chenxi. Experimental investigation of earthquake response reduction of buildings with added two types of SMA passive energy dissipation devices[J]. Earthq. Eng. Eng. Vibrat., 2003， 23(1): 133-139.

[5] 陈金金, 李爱群, 丁幼亮, 等. 空间网架结构形状记忆合 金隔震研究[J]. 工程力学, 2010, 27(9): 86-93.

CHEN Xin, LI Aiqun, DING Youliang, et al. Study on isolation of space grid structure using shape memory alloy[J]. Eng. Mech., 2010, 27(9): 86-93.

[6] 任文杰, 李宏男, 宋钢兵, 等. 新型自复位 SMA 阻尼 器对框架结构减震控制的研究 [J]. 土木工程学报, 2013(6): 14-20.

REN Wenjie, LI Hongnan, SONG Gangbing, et al. Study on seismic response control of frame structure using innovative re-centring[J] SMA damper. Chin. Civ. Eng. J., 2013(6): 14-20.

[7] KOH J S, CHO K J. Omega-shaped inchworm-inspired crawling robot with large-index-and-pitch(LIP) SMA spring actuators[J]. IEEE-ASME T. Mech., 2013， 18(2): 419-429.

[8] FRANCESCO B, MOHAMMAD E, PAOLA F, et al. A passive magneto-thermo-mechanical coupling actuated by SMA springs and MR fluid[J]. Iint. J. Struct. Stab. Dyn., 2014, 14(8): 1463-1482.

[9] SPEICHER M, HODGSON D E, DESROCHES R, et al. Shape memory alloy tension/compression device for seismic retrofit of buildings[J]. J. Mater. Eng. Perform., 2009, 18(5-6): 746-753.

[10] 蔡锦荣, 刘树堂. SMA 弹簧-轴承滚动隔震输电塔的地 震反应分析 [J]. 广东土木与建筑, 2009(5): 14-17.

CAI Jinrong, LIU Shutang. Seismic response analysis of transmission tower with SMA spring-bearing $[\mathrm{J}]$. Guangdong Archit. Civ. Eng., 2009(5): 14-17.

[11] 刘爱荣, 潘亦苏, 周本宽. 形状记忆合金弹簧变形分析 及其在振动控制中的应用 $[\mathrm{J}]$. 西南交通大学学报, 2000, 35(1): 81-85

LIU Airong, PAN Yisu, ZHOU Benkuan. Deformation analysis of SMA springs and its application to vibration control[J]. J. Southwest. Jiaotong. Univ.，2000，35(1): 81-85.

[12] 贺志荣, 张永宏, 王永善, 等. Ti49.4Ni50.6 超弹性弹 簧的相变和形变特性[J]. 金属学报, 2004, 40(1): 46-50. HE Zhirong, ZHANG Yonghong, WANG Yongshan, et al. Transformation and deformation characteristics of Ti49.4Ni50.6 superelastic spring[J]. Acta Metall. Sin., 2004, 40(1): 46-50.

[13] TOI Y, LEE J B, TAYA M. Finite element analysis of superelastic, large deformation behavior of shape memory alloy helical springs[J]. Comput. Struct., 2004, 82(20-21): 1685-1693.

[14] MIRZAEIFAR R, DESROCHES R, YAVARI A. A combined analytical, numerical, and experimental study of shape-memory-alloy helical springs[J]. Int. J. Solids Struct., 2011, 48(3-4): 611-624.

[15] AGUIAR R A A, SAVI M A, PACHECO P M C L. Experimental and numerical investigations of shape 
memory alloy helical springs[J]. Smart Mater. Struct., 2010, 19(19): 025008.

[16] HEIDARI B, KADKHODAEI M, BARATI M, et al. Fabrication and modeling of shape memory alloy springs[J]. Smart Mater. Struct., 2016， 25(12): 125003.

[17] 庄鹏, 聂攀, 薛素铎, 等. 大尺寸超弹性镍钛形状记忆 合金螺旋弹簧滞回性能 $[\mathrm{J}]$. 建筑科学与工程学报, 2016, 33(2): 114-120.

ZHUANG Peng, NIE Pan, XUE Suduo, et al. Hysteretic performance of large scale superelastic NITI shape memory alloy helical spring[J]. J. Archit. Civil Eng., 2016, 33(2): 114-120.

[18] 庄鹏, 薛素铎, 韩沝, 等. 超弹性形状记忆合金螺旋弹 簧恢复力的数值模拟 [J]. 世界地震工程, 2016(1): 237-244.

ZHUANG Peng, XUE Suduo, HAN Miao, et al. Numerical simulation of restoring force of superelastic shape memory alloy helical spring[J]. World Earthq. Eng., 2016(1): 237-244.

[19] TANAKA K, KOBAYASHI S, SATO Y. Thermomechanics of transformation pseudoelasticity and shape memory effect in alloys[J]. Int. J. Plast., 1986, 2(1): 59-72.

[20] BRINSON L C. One-dimensional constitutive behavior of shape memory alloys: Thermomechanical derivation with non-constant material functions and redefined martensite internal variable[J]. J. Mech. Phy. Solids. 1993, 2(2): 229-242.
[21] 周博, 王振清, 梁文彦. 形状记忆合金的细观力学本构 模型 $[J]$. 金属学报，2006，42(9): 919-924.

ZHOU Bo, WANG Zhenqing, LIANG Wenyan. A micromechanical constitutive model of shape memory alloys [J]. Acta Metall. Sin., 2006， 42(9): 919-924.

[22] 周博, 刘彦菊, 冷劲松, 等. 形状记忆合金的宏观力学 本构模型 $[J]$. 中国科学 G 辑, 2009, 52(9): 998-1006. ZHOU Bo, LIU Yanju, LENG Jinsong, et al. A macro-mechanical constitutive model of shape memory alloys[J]. Sci. Chin. Ser. G., 2009, 52(9): 998-1006.

[23] ZHOU B, YOON S H, LENG J S. A three-dimensional constitutive model for shape memory alloy[J]. Smart. Mater. Struct., 2009, 18(9): 095016.

[24] LAGOUDAS D, HARTL D, CHEMISKY Y, et al. Constitutive model for the numerical analysis of phase transformation in polycrystalline shape memory alloys[J]. Int. J. Plast., 2012， s 32-33(2): 155-183.

[25] RIZZONI R, MARFIA S. A thermodynamical formulation for the constitutive modeling of a shape memory alloy with two martensite phases[J]. Meccanica, 2015, 50(4): 1121-1145.

作者简介: 周博(通信作者), 男, 1972 年出生, 教授, 博士研究生导师。 主要研究方向为智能材料与结构、油气工程力学、微尺度材料力学等。 E-mail: zhoubo@upc.edu.cn 王志勇, 男, 1992 年出生。主要研究方向为智能材料与结构、微尺度材 料力学等。

薛世峰, 男, 1963 年出生, 教授, 博士研究生导师。主要研究方向为断 裂力学、油气工程力学、计算力学等。 